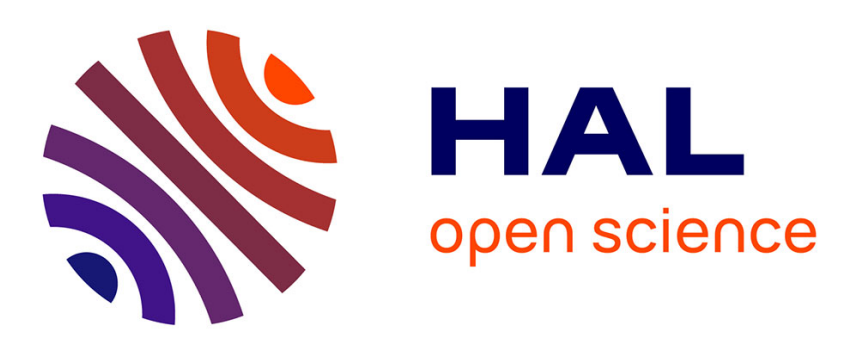

\title{
Renal Cell Carcinoma Classification from Vascular Morphology
}

Rudan Xiao, Eric Debreuve, Damien Ambrosetti, Xavier Descombes

\section{To cite this version:}

Rudan Xiao, Eric Debreuve, Damien Ambrosetti, Xavier Descombes. Renal Cell Carcinoma Classification from Vascular Morphology. MICCAI 2021 - 24th International Conference on Medical Image Computing and Computer Assisted Intervention, Sep 2021, Strasbourg, France. pp.611-621, 10.1007/978-3-030-87231-1_59. hal-03449971

\section{HAL Id: hal-03449971 https://hal.science/hal-03449971}

Submitted on 26 Nov 2021

HAL is a multi-disciplinary open access archive for the deposit and dissemination of scientific research documents, whether they are published or not. The documents may come from teaching and research institutions in France or abroad, or from public or private research centers.
L'archive ouverte pluridisciplinaire HAL, est destinée au dépôt et à la diffusion de documents scientifiques de niveau recherche, publiés ou non, émanant des établissements d'enseignement et de recherche français ou étrangers, des laboratoires publics ou privés. 


\title{
Renal Cell Carcinoma Classification from Vascular Morphology
}

\author{
Rudan Xiao (1), Eric Debreuve (2), Damien Ambrosetti (3), Xavier Descombes (1) \\ (1) Université Côte d'Azur, Inria, CNRS, Laboratoire I3S, Sophia Antipolis, France. \\ (2) Université Côte d'Azur, CNRS, Inria, Laboratoire I3S, Sophia Antipolis, France. \\ (3) CHU de Nice, Hôpital Pasteur, Laboratoire Central d'Anatomo Pathologie, France.
}

\begin{abstract}
Renal Cell Carcinoma (RCC) is one of the most common malignancies, and pathological diagnosis is the most reliable RCC diagnostic method. Recognizing the type of RCC tumor and the possibility of cell migration highly depends on the geometric and topological properties of the vascular network. Motivated by the diagnosis pipeline, we explore the use of the vascular network from the RCC histopathological image to boost the RCC classification result. To realize this, we firstly build a new vascular network-based RCC histopathological image dataset, namely VRCC200, with 200 well-labeled vascular network annotations. Based on these vascular networks of RCC histopathological images, we propose new hand-craft features, namely skeleton feature and lattice feature. These features well represent the geometric and topological properties of the vascular networks of RCC histopathological images. Then we build strong benchmark results with various algorithms (both traditional and deep learning models) on the VRCC200 dataset. The result of lattice features can beat the popular deep learning models with other features. Finally, we proved the robustness and advantage of our proposed features on a more patients' dataset VRCC60. All of the results of our experiments prove that the vascular network structure of RCC is one of the most important biomarkers for RCC diagnosis.
\end{abstract}

Keywords: RCC Histopathological Image Dataset - Vascular Network - Skeleton Feature · Lattice Feature · RCC Classification

\section{Introduction}

RCC is a highly malignant tumor in the urinary system, $90 \%$ of kidney cancers are RCC [15], which is mainly divided into clear cell RCC [18] (ccRCC) accounting for $75 \%$ of RCC and papillary RCC (pRCC) accounting for $10 \%$ of RCC. However, RCC classification is a challenging task. Cell morphology, tumor architecture, phenotype and genetics data are mainly used to define the tumor subtype. Most of the current classification research is focused on the search for biological biomarkers, aiming to define RCC subtype and also predict behavior of the tumor [8]. However, the vascular structure of these two kinds of RCCs are different and may lead to an accurate diagnosis. For example, ccRCC is characterized by a fishnet-like vascular architecture while the pRCC has a tree-like structure [34]. As shown in Fig. 2, the ccRCC vascular networks are denser and contain more junctions inside vascular networks. 
If vascular network structure is crucial and essential for the machine learningbased RCC classification is still an open question. In this paper, we explore the importance of the vascular network in the RCC diagnosis. Due to there is no public RCC dataset with vascular network annotations, we build the VRCC200 dataset with vascular annotations. VRCC200 is extracted from our larger RCC histopathological image dataset (BigRCC), which is labeled with RCC categories. The larger dataset contains the data of 158 Whole Slide Images (WSIs), coming from 68 patients, which can be cropped into 39986 patch images of ccRCC and 18254 patch images of pRCC respectively. VRCC200 has 200 vascular network segment images of ccRCC and pRCC, coming from 7 patients.

To further explore the potential of traditional algorithms, we propose two sets of features, defined "skeleton features" and "lattice features", which are extracted from the vascular network. Specifically, we compute the skeleton of the vascular network. The skeleton is a structure that embeds the topological properties of the vascular network, as shown in Fig. 2. It is composed of 3 types of elements: junction, non-end branch, end branch. Firstly, we define some meaningful features from the skeleton to form the skeleton features, containing small ending branch (NE), long NE, and Density, etc., as shown in Fig. 3. Then, we perform a series of operations on the skeleton to obtain the lattice spatial map. The lattice features that represent the regions between vessels are extracted from this spatial map. Lattice features include a set of features, such as the mean area, median area, etc. in Tab. 2.

Finally, we build solid benchmark results of traditional and deep learning methods on the VRCC200 dataset. The results of traditional algorithms with our skeleton and lattice features can beat the results of popular deep learning models (Graph Convolutional Network (GCN) [32], Convolutional Neural Network $(\mathrm{CNN})[14,24])$. Then we do testing on a new vascular annotated dataset named VRCC60, which contains 60 vascular annotated patch images, coming from 20 patients, our skeleton and lattice features still perform best. This indicates our features are robust and can embed sufficient information to characterize RCC subtypes. Also, we show the first work using GCN [32,5] with vascular graph feature [10] for the RCC histopathological images classification.

All in all, our contribution can be summarized as follows:

- We are the first work to investigate the importance of geometric and topological properties of the vascular network for RCC classification.

- We build new vascular annotated datasets, "VRCC200" and "VRCC60", for RCC histopathological image classification.

- We propose two sets of new hand-craft features, "skeleton and lattice features" to represent the vascular network, which are extracted from the vascular network of RCC histopathological images.

- We are the first to apply GCN with vascular graph features for the RCC classification task.

- We build benchmark results of various algorithms on the VRCC200 and test them on the VRCC60. 


\section{Related works}

\subsection{Histopathological Dataset}

For the histopathological classification, there are some public and popular histopathological datasets, such as CAMELYON [17] and BreakHis [27] for breast cancer, LC25000 [2] for lung and colon, but there is still a lack of accessible RCC histopathological datasets, especially with vascular network annotations. Therefore, we have built our own annotated database.

For RCC, [13] proposed an RCC dataset for RCC subtyping. [7] proposed a dataset for classifying TFE3-RCC and ccRCC. But the raw data of them is still not available.

In this paper, we focus on the RCC classification task with the vascular network, as far as we know, we are the first to realize RCC histopathological classification on RCC dataset with vascular network annotations.

\subsection{Histopathological Medical Imaging Classification}

Although the deep learning methods have achieved great progress for medical imaging tasks with the deep features $[32,5]$, the hand-craft feature still shows its robustness and efficiency in medical imaging task because of the lack of large scale datasets.

Deep learning-based on methods in medical imaging include data augmentation, network structure, unsupervised methods. Firstly, Xue et al. [31] proposed the HistoGAN for generating synthesizing imaging patches. Spanhol et al. [28] focused on extracting imaging patches from high-resolution histopathology images. Secondly, Wang et al. [29] proposed to use the Bilinear CNN for this task. Moreover, unsupervised methods also attracted more and more attention, Ren et al. [23] introduced the unsupervised domain adaption method. Peng et al. [20] developed a multi-task deep learning framework. Different from them, we use the vascular network in images and corresponding graph features, GCN [32] to solve this task, with better explainability.

To define hand-craft features in medical imaging, the main challenge is to maximize the embedded information extracted from histopathology images based on cell or nuclei (number, size or shape), tissue structure. To solve the histopathology classification, Zubiolo et al. [34] proposed to use hand-craft features (number of end nodes (NE) \& junctions (NJ), the length of end branches (LE) \& nonend branches (LJ) and their ratios) to represent the vascular network of RCC. Qureshi et al. [21] proposed subband texture features and micro-texture features. Raza et al. [22] proposed a bag-of-features framework. To further explore the potential of hand-craft features, we propose two novel hand-craft features, i.e. skeleton features based on [34] and lattice features. Different from the previous, these features are build based on the vascular networks of RCC histopathological images. 


\section{Dataset}

\subsection{Dataset Building}

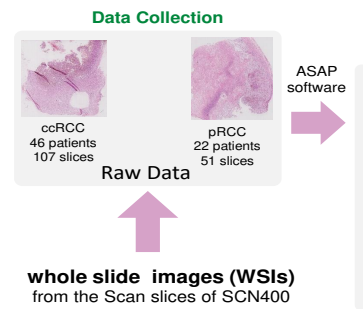

Get Patch Images

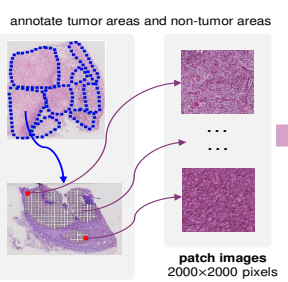

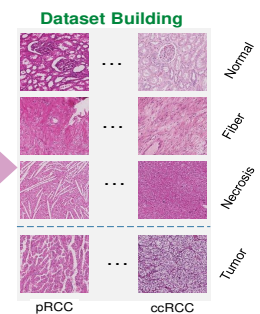

Fig. 1. Pipeline of BigRCC dataset building.

The original WSIs are Hematoxylin Eosin-stained, scanned by SCN400 scanner at 40x magnification. ccRCC includes 46 patients and a total of 107 slices, while pRCC includes 22 patients and a total of 51 slices. Tumor and non-tumor (necrosis, fiber, and normal) areas were annotated using the open-source software ASAP and stored in XML format file. Next, we cut the WSIs images into smaller histopathologic patch images $(2000 \times 2000$ Pixels $)$. All the patch images form the BigRCC dataset. As shown in Tab. 1, we analyze the statistical distribution of the BigRCC dataset.

\begin{tabular}{c|c|c|c|c|c}
\hline BigRCC dataset & Necrosis & Fiber & Normal & Tumor & Total \\
\hline ccRCC & 3324 & 1941 & 7459 & 27287 & 39986 \\
\hline pRCC & 1602 & 920 & 2105 & 13637 & 18254 \\
\hline
\end{tabular}

Table 1. The number of patch images of each category in BigRCC.

\section{$3.2 \quad$ VRCC200}

We annotated 200 tumor patch images of ccRCC and pRCC to construct the VRCC200 dataset. We use "ImageJ" software to annotate the vascular networks. As shown in Fig. 2, the vascular structure of ccRCC is like "fishnet", while pRCC is looks like "tree". To describe the vascular network we consider junctions, end branches and non-end branches (branches between two junctions).
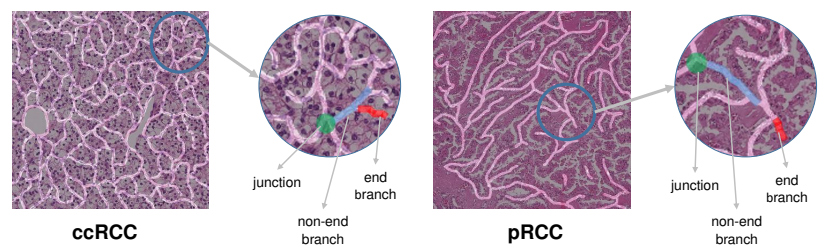

Fig. 2. The vascular network examples of ccRCC and pRCC images. 


\section{Vascular Network Feature}

\subsection{Hand-craf Features}

\begin{tabular}{|c|c|c|c|}
\hline Skleton & note & Lattice & note \\
\hline $\begin{array}{c}\mathrm{NE} \\
\text { small NE }\end{array}$ & $\begin{array}{l}\text { The Number of End branches } \\
\text { NE that }\end{array}$ & $\begin{array}{l}\text { Mean Area } \\
\text { Median Area }\end{array}$ & $\begin{array}{l}\text { Mean of all lattice areas } \\
\text { Median of all lattice areas }\end{array}$ \\
\hline $\begin{array}{l}\text { small NE } \\
\text { long NE }\end{array}$ & NE that LE more than mean of nuclear size $\times 10$ & $\begin{array}{l}\text { Median Area } \\
\text { Mean Perimeter }\end{array}$ & Mean of all lattice Perimeters \\
\hline NJ & The Number of Junctions & Median Perimeter & Median of all lattice Perimeters \\
\hline LE & The average Length of the End branches & Mean Eccentricity & Mean of all lattice Eccentricities \\
\hline $\begin{array}{c}\text { LJ } \\
\text { nonsity }\end{array}$ & $\begin{array}{l}\text { The average Length of the non-End branches } \\
\text { Sum of skeleton pixels }\end{array}$ & Median Eccentricity & Mean of all lattice Eccentricities \\
\hline $\begin{array}{l}\text { NE/NJ } \\
\text { Nenstry }\end{array}$ & $\begin{array}{c}\text { Sum of skeleton pixels } \\
\text { NE/NJ Ratio }\end{array}$ & & \\
\hline $\mathrm{LE} / \mathrm{LJ}$ & LE/LJ Ratio & & \\
\hline $\mathrm{NE} /(\mathrm{L} J+\mathrm{LE})$ & $\mathrm{NE} /(\mathrm{LJ}+\mathrm{LE})$ Ratio & & \\
\hline $\mathrm{NJ} /(\mathrm{LJ}+\mathrm{LE})$ & $\mathrm{NJ} /(\mathrm{LJ}+\mathrm{LE})$ Ratio & & \\
\hline $\mathrm{LJ} /(\mathrm{LJ}+\mathrm{LE})$ & $\mathrm{LJ} /(\mathrm{LJ}+\mathrm{LE})$ Ratio & & \\
\hline
\end{tabular}

Table 2. The explanation of every skeleton and lattice feature.

Skeleton Features. As shown in Fig. 2, the fishnet has more junctions, more non-end branches, the tree has more end branches. The graph visualization on Fig. 5 also supports our assumption. We use NE, small NE (less than nuclear size $\times 10$ ), long NE (more than nuclear size $\times 10$ ), NJ, LE, LJ, Density as the basic features of the skeleton. Moreover, we also consider NE/NJ, NE/(LJ+LE), $\mathrm{NJ} /(\mathrm{LJ}+\mathrm{LE}), \mathrm{LE} / \mathrm{LJ}, \mathrm{LJ} /(\mathrm{LJ}+\mathrm{LE})$ to make the skeleton features robust and comprehensive. The details are shown on Tab. 2 and Fig. 3.

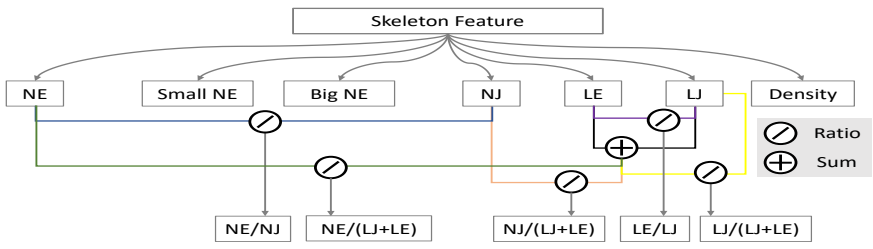

Fig. 3. Skeleton feature.

Lattice Features. As shown above, the vascular networks of ccRCC and pRCC have different structures. We propose a new set of features "lattice features" to represent the vascular network. But different from the skeleton, they are not extracted from the skeleton directly. As shown in Fig. 4, we first compute the watershed [26] on the vascular skeleton to obtain closed areas. Then we use the minima imposition method $[1,19]$ to solve the problem of local minimum and modify the distance transformation to obtain the lattice spatial map. Finally, we removing the surrounding lattices which do not include complete vascular information. Next, we define and extract 6 features from the lattice map. including Mean Area, Median Area, Mean Perimeter, Median Perimeter, Mean Eccentricity, and Median Eccentricity. The details are shown in Tab. 2.

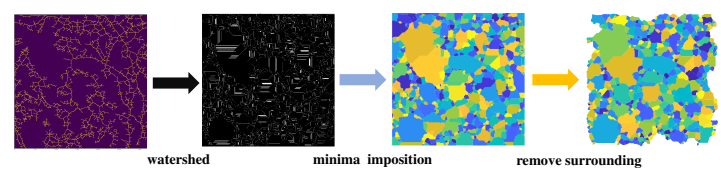

Fig. 4. The pipeline of obtaining the lattice features. 


\subsection{Deep Learning Feature}

Deep Features. Besides the hand-craft features with traditional methods, we also use the deep learning algorithm for the RCC classification. To further evaluate the importance of the vascular network, we consider both raw RCC images and vascular segment mask images as inputs, then learn these deep features with popular CNNs as the baseline experiments, such as ResNet [14], VGG Net [24], ShuffeNet [33] and Stochastic Depth Network [16].

Graph Features. Because the vascular network is a kind of graph-like structure, we transform the vascular network into graph with SKL-Graph [10], as shown in Fig. 5. Then we feed the adjacency matrix of the graph into GCN. We are the first to apply this pipeline for RCC classification. Although we only consider topological information as the branch length is not coded in the adjacency matrix, this feature is more explainable than the deep features which are black boxes.
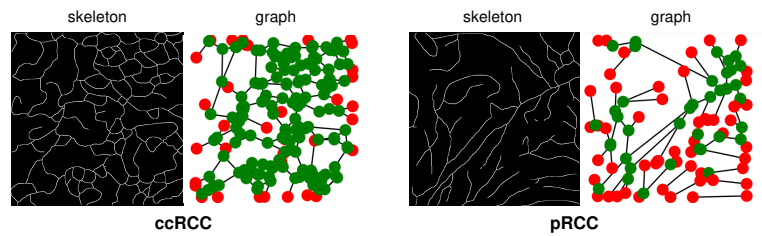

Fig. 5. The graph features of ccRCC and pRCC, red point represent end point of the vascular network, green point represent junction of the vascular network.

\section{Experiments}

\subsection{Skeleton Features and Lattice Features Analysis}

Non-parametric tests. Due to the value of the proposed features isn't normal distribution and variance heterogeneity, we use 3 non-parametric tests methods to calculate the statistical significance between the ccRCC and pRCC features. As shown in Tab. 3, there are only 5 features whose P-value is larger than 0.05 for at least one statistical test (italics). Finally, we choose the 13 features that are significant in all 3 tests. i.e. these 13 features are more suitable for classifying the ccRCC and pRCC images.

Performance on traditional algorithms. To prove the robustness and effect of skeleton and lattice features, we compare them with the baseline features: NE, NJ, LE, LJ, NE/NJ and LE/LJ [34]. As shown in Tab. 4, the proposed features, particularly after filtering by non-parameteric tests achieves much higher accuracy on almost all traditional algorithm models. This proved that our proposed features are more robust and advanced for RCC classification. 


\begin{tabular}{c|c|c|c}
\hline Features & $\begin{array}{c}\text { Mann-Whitney } \\
\text { U Test }\end{array}$ & $\begin{array}{c}\text { Moses extreme } \\
\text { reactions Test }\end{array}$ & $\begin{array}{c}\text { Kolmogorov- } \\
\text { Smirnov Z Test }\end{array}$ \\
\hline NE & $1.73 \mathrm{E}-19$ & $1.23 \mathrm{E}-13$ & $7.01 \mathrm{E}-16$ \\
small NE & $2.09 \mathrm{E}-01$ & 0.000139 & 0.083674 \\
long NE & $2.89 \mathrm{E}-21$ & $0.00 \mathrm{E}+00$ & $4.57 \mathrm{E}-18$ \\
NJ & $1.17 \mathrm{E}-07$ & 0.002281 & 0.000016 \\
LE & $1.16 \mathrm{E}-13$ & 0.007344 & $3.46 \mathrm{E}-14$ \\
LJ & $2.50 \mathrm{E}-24$ & $8.41 \mathrm{E}-19$ & $5.26 \mathrm{E}-23$ \\
density & $7.93 \mathrm{E}-29$ & $0.00 \mathrm{E}+00$ & $1.98 \mathrm{E}-27$ \\
NE/NJ & $4.76 \mathrm{E}-28$ & $2.83 \mathrm{E}-21$ & $5.25 \mathrm{E}-26$ \\
LE/LJ & 0.046331 & 1 & 0.026055 \\
NE/(LJ+LE) & 0.026 & 0.98752 & $3.45 \mathrm{E}-03$ \\
NJ/(LJ+LE) & $1.59 \mathrm{E}-28$ & $0.00 \mathrm{E}+00$ & $3.93 \mathrm{E}-27$ \\
LJ/(LJ+LE) & 0.04442 & 1 & 0.027041 \\
\hline mean_Area & $3.96 \mathrm{E}-29$ & $0.00 \mathrm{E}+00$ & $3.96 \mathrm{E}-29$ \\
median_Area & $2.65 \mathrm{E}-22$ & $5.91 \mathrm{E}-20$ & $5.25 \mathrm{E}-26$ \\
mean_Perimeter & $4.44 \mathrm{E}-08$ & 0.317547 & $1.26 \mathrm{E}-07$ \\
median_Perimeter & $2.45 \mathrm{E}-23$ & $5.91 \mathrm{E}-20$ & $5.25 \mathrm{E}-26$ \\
mean_Eccentricity & 0.003825 & 0.000006 & 0.000043 \\
median_Eccentricity & 0.048702 & 0.000139 & 0.001597 \\
\hline
\end{tabular}

Table 3. Pvalue of non-parametric tests of every feature.

\begin{tabular}{c|c|c|c}
\hline Methods & baseline & full & filtered \\
& Skeleton & Skeleton \& Lattice & Skeleton \& Lattice \\
\hline Adaboost [11] & $\mathbf{0 . 9 7 5}$ & 0.940 & 0.940 \\
DecisionTree [4] & 0.955 & $\mathbf{0 . 9 6 5}$ & $\mathbf{0 . 9 6 5}$ \\
Gradient Boosting Tree [12] & 0.955 & $\mathbf{0 . 9 6 5}$ & $\mathbf{0 . 9 6 5}$ \\
KNN [9] & 0.905 & 0.910 & $\mathbf{0 . 9 3 5}$ \\
Logistic Regression [30] & 0.970 & $\mathbf{0 . 9 8 5}$ & $\mathbf{0 . 9 8 5}$ \\
Random Forest [3] & 0.960 & 0.960 & $\mathbf{0 . 9 6 5}$ \\
SVM RBF [6] & 0.965 & 0.980 & $\mathbf{0 . 9 8 5}$ \\
SVM Sigmoid [25] & 0.790 & $\mathbf{0 . 9 6 5}$ & $\mathbf{0 . 9 6 5}$ \\
\hline
\end{tabular}

Table 4. The Accuracy result of 3 features on different algorithms. "Baseline Skeleton" refers to previous skeleton feature [34], "full Skeleton \& Lattice" refers to all the skeleton and lattice feature we proposed, "filtered Skeleton \& Lattice" refers to a part of skeleton and lattice feature whose features are filtered by non-parametric tests.

\subsection{Vascular-based RCC Classification Benchmark}

In this section, we conduct abundant experiments on the VRCC200 dataset with traditional and deep learning models. The accuracy results of validation are the average of leave-one-out cross-validation. There are 7 patients' data, the data of 1 patient is used for testing each time. To check the robustness of the models, we annotated more patients' dataset VRCC60 for testing. VRCC60 dataset has a total of 60 patch images, coming from 20 patients (10 for ccRCC, 10 for pRCC).

Validation results on VRCC200. As shown in Tab. 5, the results of the traditional methods range from $94 \%$ to $98.5 \%$, which is higher than most others. This demonstrates that the proposed skeleton and lattice features are advanced. 
8

Paper ID 2045

\begin{tabular}{c|c|c|c|c}
\hline Methods & Input & Feature & Acc(val) & Acc(test) \\
\hline Traditional Algorithm & \multicolumn{4}{|c}{} \\
\hline Adaboost [11] & Segment & filtered Skeleton \& Lattice & $\mathbf{0 . 9 4 0}$ & 0.935 \\
DecisionTree [4] & Segment & filtered Skeleton \& Lattice & $\mathbf{0 . 9 6 5}$ & 0.935 \\
Gradient Boosting Tree [12] & Segment & filtered Skeleton \& Lattice & $\mathbf{0 . 9 6 5}$ & $\mathbf{0 . 9 6 5}$ \\
KNN [9] & Segment & filtered Skeleton \& Lattice & $\mathbf{0 . 9 3 5}$ & 0.915 \\
Logistic Regression [30] & Segment & filtered Skeleton \& Lattice & $\mathbf{0 . 9 8 5}$ & 0.915 \\
Random Forest [3] & Segment & filtered Skeleton \& Lattice & 0.965 & $\mathbf{0 . 9 8 5}$ \\
SVM RBF [6] & Segment & filtered Skeleton \& Lattice & $\mathbf{0 . 9 8 5}$ & 0.915 \\
SVM Sigmoid [25] & Segment & filtered Skeleton \& Lattice & $\mathbf{0 . 9 6 5}$ & 0.865 \\
\hline Deep Learning & \multicolumn{3}{|c|}{} \\
\hline ResNet-18 [14] & Raw & Deep & 0.817 & $\mathbf{0 . 8 6 7}$ \\
VGG-16 [24] & Raw & Deep & $\mathbf{0 . 9 0 8}$ & 0.800 \\
ShuffleNet_V1 [33] & Raw & Deep & $\mathbf{0 . 8 6 7}$ & 0.800 \\
Stochastic Depth [16] & Raw & Deep & $\mathbf{0 . 8 6 9}$ & 0.817 \\
\hline ResNet-18 [14] & Segment & Deep & 0.937 & 0.917 \\
VGG-16 [24] & Segment & Deep & 0.883 \\
ShuffleNet_V1 [33] & Segment & Deep & $\mathbf{0 . 8 8 7}$ & 0.867 \\
Stochastic Depth [16] & Segment & Deep & $\mathbf{0 . 9 7 9}$ & 0.933 \\
\hline GCN+SAGPoolg [32] & Segment & Graph & $\mathbf{0 . 9 6 2}$ & 0.833 \\
GCN+SAGPoolh [5] & Segment & Graph & $\mathbf{0 . 8 6 6}$ & 0.817 \\
\hline
\end{tabular}

Table 5. The benchmark results on the VRCC200. Segment images are vascular network segment masks. Acc (val) is the accuracy result of "leave one out" crossvalidation on VRCC200. Acc (test) is the accuracy result of testing on the VRCC60.

For the deep learning-based methods, the accuracy with vascular segment input is much better than that with raw image input, which indicates the importance of vascular network again. We argue that only using the segments of the vascular network may suppress background noise, resulting in higher accuracy.

Also, we obtain results of two GCN models. They're lower than some vascular segmentation input deep learning model (97.9\% VS 96.2\%) and traditional algorithm models (98.5\% VS 96.2\%). May because the graph feature only contains the topological information of vascular, but other features contain both topological and geometrical information.

Testing results on VRCC60. Compare with the validation result on VRCC200, most of the accuracy has declined, but it can still maintain above $80.0 \%$. Moreover, skeleton and lattice features are still the best performers (86.5\% to $98.5 \%)$. This demonstrates that our proposed features are robust on more patients' data.

\section{Conclusion}

In this paper, we build VRCC200 and VRCC60 datasets to experiment. We can characterize ccRCC and pRCC only from vascular properties. The result of our proposed "skeleton and lattice features" (contain both topological and geometrical information) better than the "graph features" (only topological information) and the baseline deep learning features. Our proposed features provide an explanation for RCC classification and robustness on more patients' data as well. 


\section{References}

1. Beucher, S.: Segmentation d'images et morphologie mathématique. Ph.D. thesis, Ecole Nationale Supérieure des Mines de Paris (1990)

2. Borkowski, A.A., Bui, M.M., Thomas, L.B., Wilson, C.P., DeLand, L.A., Mastorides, S.M.: Lung and colon cancer histopathological image dataset (lc25000). $\operatorname{arXiv}(2019)$

3. Breiman, L.: Random forests. Machine learning 45(1), 5-32 (2001)

4. Breiman, L., Friedman, J.H., Olshen, R.A., Stone, C.J.: Classification and regression trees. The Wadsworth Brooks/Cole statistics/probability series, Wadsworth Brooks/Cole Advanced Books Software, Monterey, CA (1984)

5. Cangea, C., Veličković, P., Jovanović, N., Kipf, T., Liò, P.: Towards sparse hierarchical graph classifiers. arXiv (2018)

6. Cao, H., Naito, T., Ninomiya, Y.: Approximate rbf kernel svm and its applications in pedestrian classification. In: MLVMA Workshop (2008)

7. Cheng, J., Han, Z., Mehra, R., Shao, W., Cheng, M., Feng, Q., Ni, D., Huang, K., Cheng, L., Zhang, J.: Computational analysis of pathological images enables a better diagnosis of tfe 3 xp11. 2 translocation renal cell carcinoma. Nature communications 11(1), 1-9 (2020)

8. Cheville, J.C., Lohse, C.M., Zincke, H., Weaver, A.L., Blute, M.L.: Comparisons of outcome and prognostic features among histologic subtypes of renal cell carcinoma. The American journal of surgical pathology 27(5), 612-624 (2003)

9. Cover, T., Hart, P.: Nearest neighbor pattern classification. IEEE transactions on information theory 13(1), 21-27 (1967)

10. Debreuve: https://gitlab.inria.fr/edebreuv/sklgraph (2020)

11. Freund, Y., Schapire, R.E.: A decision-theoretic generalization of on-line learning and an application to boosting. Journal of computer and system sciences 55(1), 119-139 (1997)

12. Friedman, J.H.: Greedy function approximation: a gradient boosting machine. Annals of statistics pp. 1189-1232 (2001)

13. Gao, Z., Puttapirat, P., Shi, J., Li, C.: Renal cell carcinoma detection and subtyping with minimal point-based annotation in whole-slide images. In: MICCAI. pp. 439448. Springer (2020)

14. He, K., Zhang, X., Ren, S., Sun, J.: Deep residual learning for image recognition. In: CVPR. pp. 770-778 (2016)

15. Hsieh, J.J., Purdue, M.P., Signoretti, S., Swanton, C., Albiges, L., Schmidinger, M., Heng, D.Y., Larkin, J., Ficarra, V.: Renal cell carcinoma. Nature reviews Disease primers 3(1), 1-19 (2017)

16. Huang, G., Sun, Y., Liu, Z., Sedra, D., Weinberger, K.Q.: Deep networks with stochastic depth. In: ECCV. pp. 646-661. Springer (2016)

17. Litjens, G., Bandi, P., Ehteshami Bejnordi, B., Geessink, O., Balkenhol, M., Bult, P., Halilovic, A., Hermsen, M., van de Loo, R., Vogels, R., et al.: 1399 h\&e-stained sentinel lymph node sections of breast cancer patients: the camelyon dataset. GigaScience $\mathbf{7}(6)$, giy065 (2018)

18. Lopez-Beltran, A., Scarpelli, M., Montironi, R., Kirkali, Z.: 2004 who classification of the renal tumors of the adults. European urology 49(5), 798-805 (2006)

19. Meyer, F., Beucher, S.: Morphological segmentation. Journal of visual communication and image representation 1(1), 21-46 (1990)

20. Peng, T., Boxberg, M., Weichert, W., Navab, N., Marr, C.: Multi-task learning of a deep k-nearest neighbour network for histopathological image classification and retrieval. In: MICCAI. pp. 676-684. Springer (2019) 
21. Qureshi, H., Sertel, O., Rajpoot, N., Wilson, R., Gurcan, M.: Adaptive discriminant wavelet packet transform and local binary patterns for meningioma subtype classification. In: MICCAI. pp. 196-204. Springer (2008)

22. Raza, S.H., Parry, R.M., Moffitt, R.A., Young, A.N., Wang, M.D.: An analysis of scale and rotation invariance in the bag-of-features method for histopathological image classification. In: MICCAI. pp. 66-74. Springer (2011)

23. Ren, J., Hacihaliloglu, I., Singer, E.A., Foran, D.J., Qi, X.: Adversarial domain adaptation for classification of prostate histopathology whole-slide images. In: MICCAI. pp. 201-209. Springer (2018)

24. Simonyan, K., Zisserman, A.: Very deep convolutional networks for large-scale image recognition. arXiv (2014)

25. Smola, A.J., Bartlett, P., Schölkopf, B., Schuurmans, D.: Probabilities for sv machines (2000)

26. Soille, P., Vincent, L.M.: Determining watersheds in digital pictures via flooding simulations. In: Visual Communications and Image Processing'90: Fifth in a Series. vol. 1360, pp. 240-250. International Society for Optics and Photonics (1990)

27. Spanhol, F.A., Oliveira, L.S., Petitjean, C., Heutte, L.: A dataset for breast cancer histopathological image classification. Ieee transactions on biomedical engineering 63(7), 1455-1462 (2015)

28. Spanhol, F.A., Oliveira, L.S., Petitjean, C., Heutte, L.: Breast cancer histopathological image classification using convolutional neural networks. In: IJCNN. pp. 2560-2567. IEEE (2016)

29. Wang, C., Shi, J., Zhang, Q., Ying, S.: Histopathological image classification with bilinear convolutional neural networks. In: EMBC. pp. 4050-4053. IEEE (2017)

30. Wright, R.E.: Logistic regression. (1995)

31. Xue, Y., Ye, J., Zhou, Q., Long, L.R., Antani, S., Xue, Z., Cornwell, C., Zaino, R., Cheng, K.C., Huang, X.: Selective synthetic augmentation with histogan for improved histopathology image classification. Medical Image Analysis 67, 101816 (2021)

32. Zhang, M., Cui, Z., Neumann, M., Chen, Y.: An end-to-end deep learning architecture for graph classification. In: AAAI. vol. 32 (2018)

33. Zhang, X., Zhou, X., Lin, M., Sun, J.: Shufflenet: An extremely efficient convolutional neural network for mobile devices. In: CVPR. pp. 6848-6856 (2018)

34. Zubiolo, A., Debreuve, E., Ambrosetti, D., Pognonec, P., Descombes, X.: Is the vascular network discriminant enough to classify renal cell carcinoma? In: CBMI. pp. 1-6. IEEE (2016) 